\title{
EL TRIBUNAL EUROPEO DE DERECHOS HUMANOS Y LA TÉCNICA DE LAS SENTENCIAS PILOTO: UNA PEQUEÑA REVOLUCIÓN EN MARCHA EN ESTRASBURGO...*
}

POR

ELISABETH LAMBERT ABDELGAWAD**

\section{CONSIDERACIONES INTRODUCTORIAS}

La saturación del Tribunal Europeo de Derechos Humanos, ya de dominio público en el seno de la comunidad de juristas, bien pudiera encontrarse en el origen de una profunda evolución, y hasta de una verdadera revolución en Estrasburgo. Antes incluso de la entrada en vigor del Protocolo núm. 14, que en principio se espera para los próximos meses (el único obstáculo actual lo representa la ratificación de la Federación Rusa), el Tribunal Europeo, ostensiblemente preocupado por

* La autora desea mostrar su sincero agradecimiento al profesor Luis Jimena Quesada (Universitat de València) por haber efectuado la traducción al español de la versión original francesa del presente artículo.

** Profesora investigadora del Centro Nacional de Investigación Científica (CNRS, Centre national de la recherche scientifique) en la Université Paris 1 Panthéon - Sorbonne (Unité mixte de recherche de droit comparé), Francia. El sitio web de la UMR de Derecho comparado puede ser visitado en la dirección siguiente: http://www.umrdc.fr. 
la supervivencia del sistema, ha iniciado, de consuno con otras instancias del Consejo de Europa $-y$ señaladamente el Comité de Ministros - la técnica denominada de las sentencias piloto desde la sentencia Broniowski contra Polonia de junio de 2004. El procedimiento de las sentencias piloto, sin embargo, no sería más que un elemento de una operación más amplia tendente a transformar en profundidad la misión del Tribunal Europeo. Para poder apreciar mejor la amplitud de esta evolución, es importante traer a colación algunos datos introductorios referentes a los efectos de las sentencias del Tribunal y al control de la ejecución de las obligaciones por parte de los Estados.

Las dos disposiciones pertinentes en materia de ejecución de sentencias son los artículos 46 y 41 del Convenio Europeo'. En el asunto Scordino contra Italia de 29 de marzo de 2006², el Tribunal Europeo «recuerda que, a tenor del artículo 46, las Altas Partes Contratantes se comprometen a dar cumplimiento a las sentencias definitivas dictadas por el Tribunal en los litigios en los que sean partes, estando encomendada al Comité de Ministros la fiscalización de la ejecución de dichas sentencias. De ello se desprende, en particular, que cuando el Tribunal constata una violación, el Estado demandado tiene la obligación jurídica, no solamente de abonar a los interesados las sumas concedidas en virtud de la compensación equitativa prevista por el artículo 41 , sino asimismo de elegir, bajo el control del Comité de Ministros, las medidas generales y/o, en su caso, individuales a incorporar a su orden jurídico interno con el fin de poner fin a la violación constatada por el Tribunal y de paliar en la medida de lo posible las consecuencias de dicha violación. El Estado demandado tiene en sus manos la posibilidad, bajo el control del Comité de Ministros, de elegir los medios de cumplir con su obligación jurídica a la vista del artículo 56 del Convenio, siempre y cuando esos medios sean compatibles con las conclusiones contenidas en el fallo del Tribunal». El contenido de la obligación de cumplir la sentencia se descompone, de tal suerte, en tres vertientes: el pago de la compensación equitativa eventualmente concedida por el Tribunal, la adopción de medidas individuales no pecu-

1 Según el artículo 46: "1. Las Altas Partes Contratantes se comprometen a acatar las sentencias definitivas del Tribunal en los litigios en que sean partes. 2. La sentencia definitiva del Tribunal será transmitida al Comité de Ministros, que velará por su ejecución". Según el artículo 41, "si el Tribunal declara que ha habido violación del Convenio o de sus Protocolos y si el Derecho interno de la Alta Parte Contratante sólo permite de manera imperfecta reparar las consecuencias de dicha violación, el Tribunal concederá a la parte perjudicada, si así procede, una satisfacción equitativa».

2 TEDH, Gran Sala, 29.3.2006, § 233. 
niarias, y/o la adopción de medidas generales, según las circunstancias de cada asunto.

Desde la puesta en marcha del sistema europeo, el Tribunal, cuyo único poder reconocido en el texto del Convenio es el de conceder eventualmente una compensación equitativa, siempre se había basado en el artículo 41 en lo atinente a los efectos de sus sentencias, rehusando así indicar las otras medidas a adoptar. Algunos incluso habían mostrado la tendencia de deducir de semejante dato que los Estados únicamente tenían la obligación de pagar la suma concedida por el Tribunal. A lo largo de los años, empero, el Tribunal se ha esforzado en afinar el poder que ostenta relativo a la concesión de la compensación equitativa, estimándose competente para fijar la moneda de pago, para fijar un plazo de tres meses para el pago y para acompañar la obligación de pago de la paralela obligación de abonar intereses de demora ${ }^{3}$. El Tribunal, al conceder casi automáticamente una compensación equitativa, ha propiciado que la doctrina se haya apresurado a criticar la deriva de mercantilización del contencioso europeo ${ }^{4}$. En paralelo, la obligación de cumplir con las sentencias ha sido siempre interpretada como una mera obligación de resultado, que dejaba libres a los Estados de elegir los medios susceptibles de ser empleados para respetar una sentencia condenatoria, considerándose válida dicha libertad «bajo el control del Comité de Ministros" y "en la medida en que dichos medios sean compatibles con las conclusiones contenidas en el fallo del Tribunal ${ }^{5}$. Ciertamente, habían sido explicitadas algunas excepciones, en particular en el contencioso referente al derecho de propiedad, pero ello sólo había comportado una alteración mínima de un principio sólidamente arraigado en la práctica.

La técnica de las sentencias piloto ha venido a alterar de plano ese cuadro tradicional. El Tribunal Europeo parece haberse atribuido nuevos poderes en virtud del artículo 46 del Convenio y parece ahora dar

${ }^{3}$ Cfr. mis trabajos: LAMBERT ABDELGAWAD, E.: Les effets des arrêts de la Cour européenne des droits de l'homme, Contribution à une approche pluraliste du droit européen des droits de l'homme, Bruylant, Bruxelles, 1999. LAMBERT ABDELGAWAD, E.: L'exécution des arrêts de la Cour européenne des droits de l'homme, Dossiers sur les droits de l'homme n. ${ }^{\circ} 19$, éd. du Conseil de l'Europe (versión inglesa: The execution of judgments of the European Court of human rights, Human

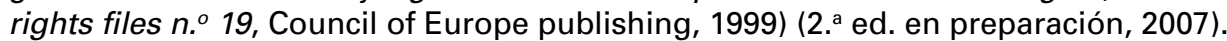

${ }^{4}$ FLAUSS, J.F.: "Le contentieux de la réparation devant la Cour européenne des droits de l'homme: eldorado pour les victimes et fonds de commerce pour les conseils?», Mélanges Jean-Pierre Sortais, Bruylant, Bruxelles, 2002, pp. 155 ss.

5 TEDH, sentencia de 22.6.2004, Broniowski contra Polonia, § 192. 
prioridad al artículo 46 con respecto al artículo 41 . Si bien es cierto que se trata de un instrumento tendente a facilitar la ejecución de las sentencias $y$, por ente, a prevenir un contencioso repetitivo, este nuevo mecanismo ante el Tribunal revela en realidad que se está sucediendo una serie de transformaciones de primer orden en el contencioso de Estrasburgo, mediante una mutación del Tribunal que viene provocada por él mismo.

\section{LA TÉCNICA DE LAS SENTENCIAS PILOTO: UN INSTRUMENTO PRIMA FACIE ANODINO TENDENTE A PREVENIR UN CONTENCIOSO REPETITIVO}

La saturación del Comité de Ministros, órgano principal encargado de la ejecución de las sentencias, es al menos tan impresionante como la padecida por el Tribunal, por un efecto de vasos comunicantes que es evidente y que sencillamente aparece desfasado en el tiempo, pero genera una situación que puede adoptar unas proporciones dramáticas en los años venideros. En la última reunión sobre derechos humanos del Comité de Ministros el 5 de diciembre de 2006, el número de asuntos pendientes, en constante progresión, se elevaba a 5522 (frente a 4322 en noviembre de 2005). El número de asuntos incluidos en el orden del día alcanzaba la cifra de 5308; el total de nuevos asuntos con motivo de esa misma reunión era del orden de 269 (frente a 165 en noviembre de 2005) ${ }^{6}$. Si la mitad de los asuntos se refiere al no pago de la compensación equitativa en el plazo fijado por el Tribunal, una parte importante de ellos tiene que ver con el incumplimiento de la adopción de las medidas generales exigidas en virtud de la obligación de no reproducción del ilícito cometido. Con ocasión de la reunión de octubre de 2006, el Comité tuvo que hacer frente a la cuestión de la adopción de medidas generales tendentes a prevenir nuevas violaciones en 186 asuntos o grupos de asuntos. Siendo consciente de que la ausencia de adopción rápida de medidas generales genera un contencioso repetitivo en Estrasburgo, el Tribunal Europeo se sintió compelido a reaccionar: con ello se adoptaba la técnica de las sentencias piloto.

6 Véase el documento CM/Del/OJ/DH(2006)982, 10.01.2007, 982. a reunión (DH), 5-6 diciembre 2006, Orden del día anotado, Estadísticas. 


\section{El asunto Broniowski contra Polonia}

El asunto Broniowski contra Polonia constituye la primera ilustración en la materia ${ }^{7}$. En dicho asunto, que versaba sobre la compatibilidad con el Convenio de una disposición legislativa que afectaba a un gran número de personas (alrededor de ochenta mil -80.000), el Tribunal vino a concluir por vez primera que existía una violación sistemática; según el Tribunal, la violación «tiene[tenía] su origen en un problema a gran escala resultante de una disfunción de la legislación polaca y de una práctica administrativa, y que ha[bía] afectado, y todavía [podía] afectar en el futuro, a un gran número de personas" ( $\S$ 189). En consecuencia, el Tribunal declaraba claramente que se imponían medidas generales a nivel nacional, medidas que debían tomar en consideración a las numerosas personas perjudicadas y que debían ser adecuadas para paliar la deficiencia estructural que se desprendía de la constatación de violación declarada por el Tribunal. Éste precisaba además que dichas medidas debían comprender un mecanismo apto para ofrecer a las personas lesionadas una reparación por la violación declarada del Convenio. A mayor abundamiento, el Tribunal decidía congelar el examen de los asuntos pendientes a la espera de la adopción de un mecanismo interno de reparación. En este primer asunto (del mismo modo que en los que se sucederán en $2005^{8}$ ), el número de asuntos pendientes constituye un criterio primordial.

7 Véase mi nota, "La Cour européenne au secours du Comité des ministres pour une meilleure exécution des arrêts pilote", Rev. trim. dr. h. (61/2005), pp. 203224.

${ }^{8}$ En el asunto Lukenda contra Eslovenia (6.10.2005, Sección tercera), 500 asuntos pendientes idénticos se encuentran registrados ante el Tribunal Europeo y son todos ellos relativos al mismo problema sistemático de la duración de los procedimientos internos. En el asunto Xenides-Arestis contra Turquía (TEDH, sentencia de 22.12.2005, Sección tercera) referente a la violación de los artículos 8 y 1 del Protocolo núm. 1, en la medida en que a los demandantes se había impedido el acceso a sus domicilios y bienes situados en la zona norte de Chipre, el Tribunal constata, bajo el ángulo del artículo 6 , que dicha declaración de violación tiene su origen en un amplio problema que afecta a un gran número de personas, y revela una política o una práctica llevada a cabo en la "República turca de Chipre del Norte». Alrededor de 1400 demandas (en esencia, formuladas por chipriotas griegos y dirigidas contra Turquía) relativas al derecho de propiedad se encuentran pendientes ante el Tribunal Europea. Así, el Tribunal requiere a Turquía para que introduzca, en el plazo de tres meses, una vía de recurso que garantice una reparación verdaderamente efectiva para la demandante, pero asimismo para el conjunto de las 1400 demandas análogas pendientes ante el Tribunal. 
El fundamento de esta nueva política debe buscarse en el uso hecho por el Tribunal del artículo 46 del Convenio, y de manera subsidiaria en la referencia a la Recomendación 2004(6) del Comité de Ministros de 12 de mayo de 2004 sobre la mejora de los recursos internos y a la Resolución de la misma fecha sobre las sentencias que revelan un problema estructural.

En el asunto Broniowski, la Gran Sala estimaba que la controversia no se planteaba bajo el ángulo del artículo 41, técnica que permite ejercer presión sobre el Estado y evaluar mejor el resto del daño potencialmente aún no reparado en el ámbito interno. Se trataba, según la sentencia de la Gran Sala de 28 de septiembre de 2005 que ratificaba la solución amistosa alcanzada en el asunto Broniowski, de una posición lógica, conforme al principio de subsidiariedad del sistema europeo, y que ofrece al Estado la posibilidad de adoptar paralelamente las medidas individuales (pecuniarias y/o extra pecuniarias) y generales que procedan ${ }^{9}$. Mediante el control operado de tal modo en vista del archivo del asunto, el Tribunal se siente compelido a evaluar la efectividad de las medidas generales e individuales exigidas a título de reparación, de la misma manera que sucede con el Comité de Ministros en virtud del artículo $46.2^{10}$. A este respecto, no parece probable que el Comité vaya a negarse a adoptar una resolución definitiva tras el satisfecit

9 TEDH, sentencia de 28.9.2005, Broniowski contra Polonia (arreglo amistoso), Gran Sala, § 36: «No cabe excluir que incluso antes de que el Estado demandado adopte una medida general, o una medida general adecuada, en ejecución de una sentencia piloto sobre el fondo (artículo 46 del Convenio), el Tribunal Europeo se vea llamado a dictar una sentencia de archivo de la demanda sobre la base de un arreglo amistoso [artículos 37.1.b) y 39], o a otorgar una satisfacción equitativa al demandante (artículo 41). Sin embargo, teniendo en cuenta el carácter sistemático o estructural del fallo que se encuentra en el origen de la constatación de violación en una sentencia piloto, es evidentemente deseable para el buen funcionamiento del mecanismo del Convenio que el restablecimiento individual y el restablecimiento general vayan de la mano. Está en manos del Estado demandado el adoptar paralelamente las medidas de carácter general y las medidas de carácter individual necesarias y el proceder a un arreglo amistoso con el demandante sobre la base de un acuerdo que integre esas dos categorías de medidas, lo que por ello mismo reforzará el carácter subsidiario del mecanismo de protección de los derechos humanos instaurado por el Convenio y facilitará al Tribunal Europeo y al Comité de Ministros el cumplimiento de las tareas que les confían respectivamente el artículo 41 y el artículo 46 del Convenio".

10 TEDH, sentencia de 28.9.2005, Broniowski contra Polonia (arreglo amistoso), Gran Sala, § 42: sin pretender prejuzgar la conclusión del Comité, el Tribunal Europeo es consciente del hecho de que su control es prácticamente idéntico al operado por el Comité sobre la base del artículo 46.2 del Convenio. 
dado por el Tribunal en su solución amistosa. Por tanto, el trabajo del Comité debería ser una simple formalidad en este tipo de asuntos. Sin embargo, el Tribunal no cuenta siempre con los medios para evaluar la efectividad de las medidas internas. En el asunto Sejdovic contra Italia de 1.3.2006, la Gran Sala, con apoyo en el precedente Broniowski y en los artículos 46 y 1 del Convenio, reconoce una "deficiencia» en el orden jurídico italiano que impide un nuevo proceso para los condenados en rebeldía. Pero, al ponderar la reforma efectuada del Código de procedimiento penal, el Tribunal no estima necesario indicar medidas generales, considerando que era demasiado pronto (en ausencia de jurisprudencia nacional) para evaluar si dicha reforma satisfacía las exigencias del Convenio ${ }^{11}$.

Teniendo en cuenta el primer éxito conocido merced al asunto Broniowski y el eco generalmente muy favorable de dicha jurisprudencia $^{12}$, el Tribunal se ha visto así animado a reiterar esa posición, incluso en otras composiciones diversas a la de la Gran Sala ${ }^{13}$. El Grupo de Sabios se ha mostrado igualmente satisfecho con el procedimiento de la "sentencia piloto» del Tribunal Europeo, propugnando que sean fijados plazos de control (§ 105) ${ }^{14}$.

\section{La desnaturalización y extensión del campo de aplicación de los asuntos pilotos}

Si en el asunto Broniowski de la Gran Sala, el fundamento de esta nueva técnica cabe hallarlo, de un lado, en el artículo 46 del Convenio y, de otro lado, en la Resolución (2004)3 del Comité sobre las sentencias que revelan un problema estructural subyacente y en la Recomendación (2004)6 sobre la mejora de los recursos internos, la Sección tercera del Tribunal, en los asuntos Lukenda y Xenides-Arestis, menciona respectivamente los artículos 46 y 1 del Convenio y sólo el artículo 46. Por lo demás, en este último asunto, ya no hay mención alguna a pro-

11 TEDH, sentencia de 10.11.2004, Sejdovic contra Italia, Sección primera.

12 Véanse, por ejemplo, las posiciones mantenidas en el Rapport Woolf : Etude des méthodes de travail, Cour européenne des droits de I'homme, diciembre 2005, The Right Honorable The Lord Woolf, p. 37: "Este procedimiento de la sentencia piloto es vital para el tratamiento de los asuntos repetitivos".

13 Bajo el ángulo del asunto Lukenda contra Eslovenia, es importante subrayar la opinión individual del juez Zagrebelsky, para quien el recurso a la técnica del asunto piloto debería ser competencia únicamente de la Gran Sala.

${ }_{14} \mathrm{CM}(2006) 203$ de 15.11.2006. 
blema estructural o sistemático, sino que se habla más bien sólo de «un amplio problema que afecta a un gran número de personas" (§ 38 ). Es verdad que el Tribunal tiene tendencia a emplear indiferentemente los términos "sistemático", "estructural» o "problema a gran escala»" ${ }^{15}$ para designar el mismo fenómeno.

Debe subrayarse asimismo que la congelación de los asuntos pendientes, que parecía ser una medida faro de la técnica de las sentencias pilotos en el primer asunto Broniowski para obligar al Estado a una ejecución "rápida", no ha sido retomada en los asuntos siguientes ${ }^{16}$. Sin embargo, en el asunto Xenides-Arestis, la Sección tercera del Tribunal ha precisado, incluso en la parte dispositiva de la sentencia, la obligación del Estado "de establecer un recurso que garantice verdaderamente una reparación efectiva de las violaciones del Convenio constatadas en el caso de autos con respecto a la pretensión principal de la parte demandante, pero también en todos los asuntos similares pendientes ante el Tribunal, según los principios de protección de los derechos enunciados en los artículos 8 del Convenio y 1 del Protocolo n. ${ }^{\circ} 1$, y de conformidad con su decisión sobre la admisibilidad de 14 de marzo de 2005». Lo más sorprendente de este asunto (lo cual no se ha reproducido hasta el presente) ${ }^{17}$ es que el Tribunal exige que dicho recurso esté "disponible dentro del plazo de tres meses a contar desde la fecha del pronunciamiento de la presente sentencia, y deberá proporcionarse una reparación en los tres meses siguientes" (párrafo 40). Semejante obligación viene confirmada por el hecho de que el Gobierno debe hacer llegar al Tribunal «informaciones detalladas sobre el recurso y su puesta a disposición» "en el plazo de tres meses a contar desde la fecha del pronunciamiento de la sentencia", y "suministrarle tres meses más tarde informaciones referentes a la reparación", tal

15 Véase, para una superposición de los tres calificativos, el asunto de la Gran Sala Scordino contra Italia de 29.03.2006, § 229.

16 Según SUNDBERG, F.: «El nuevo enfoque de 'congelación' crea sin embargo una situación de no-derecho (...)». Esa congelación "podría asimismo, en situaciones complejas, ser un handicap para la ejecución, en la medida en que las autoridades nacionales podrían experimentar dificultades para identificar las medidas de ejecución apropiadas sin más indicaciones por parte del Tribunal Europeo a través del examen de los otros asuntos" ("L'effectivité des recours internes suite à des arrêts pilote", in Cohen-Jonathan, G., Flauss, J. F., et Lambert Abdelgawad, E. (dir.): De l'effectivité des recours internes dans I'application de la Convention européenne des droits de I'homme, Bruylant, Nemesis, Coll. Droit et Justice n. ${ }^{\circ} 69,2006$, pp. 259275, pp. 262-263).

17 En el asunto Lukenda, se precisa en la parte dispositiva que las medidas deben ser adoptadas "dentro de un plazo razonable» ("within reasonable time»). 
como se ordena en el fallo. Por último, la obligación de establecer recursos efectivos, una de las características primordiales de las sentencias piloto, debería aligerar considerablemente la carga de trabajo del Tribunal y del Comité de Ministros.

El año 2006 se ha saldado con una extensión del campo de aplicación del recurso a dicha técnica. En efecto, el criterio del número de asuntos repetitivos pendientes se ha convertido en secundario, incluso insignificante; el Tribunal otorga en la práctica importancia al (solo) hecho de que otros asuntos que planteen el mismo problema general pudieren potencialmente llegar a Estrasburgo. En el asunto Scordino contra Italia ${ }^{18}$, relativo a la indemnización por una expropiación, el Tribunal señala que la constatación de violación del derecho de propiedad de los demandantes "(...) tiene su origen en un problema a gran escala que resulta de una disfunción de la legislación italiana, y que ha afectado, pudiendo todavía seguir afectando en el futuro, a un gran número de personas". El Tribunal evoca ciertamente un problema estructural (no reconocido por las autoridades nacionales, salvo por la Corte Constitucional en decisiones de 1983 y 1993) respecto del cual, aquél reconoce que solamente "algunas decenas de demandas" se encuentran en estado de litispendencia ante él. La Gran Sala recurre a los fundamentos del artículo 46 del Convenio, de la Resolución (2004)3 y de la Recomendación (2004)6 del Comité, así como al artículo 1 del Convenio. Así, el Tribunal requiere al Estado para que adopte medidas generales, respecto de las cuales el propio Tribunal introduce precisiones ${ }^{19}$.

18 TEDH, sentencia de 29.3.2006, Gran Sala. Así como todos los demás asuntos sustanciados el mismo día ante la Gran Sala contra Italia (únicamente para la cuestión de la duración excesiva de los procedimientos) [Cocchiarella contra Italia; Giuseppe Mostacciuolo contra Italia (n. ${ }^{\circ}$ 1); Musci contra Italia; Giuseppina y Orestina Procaccini contra Italia; Riccardi Pizzati contra Italia; Ernestina Zullo contra Italia; Apicella contra Italia: "124. Ante el Tribunal se encuentran actualmente pendientes centenares de asuntos que versan sobre las indemnizaciones otorgadas por tribunales de apelación en el marco de procedimientos 'Pinto' antes del giro jurisprudencial de la Corte de Casación y/o la demora en el pago de las sumas en cuestión»]. Véase asimismo la sentencia de la Sección cuarta, Stornaiuolo contra Italia, de 8.8.2006 sobre los dos aspectos (indemnización de expropiación y duración excesiva de los procedimientos), que es una reproducción de Scordino.

19 «Por tanto, semejantes medidas deben comprender un mecanismo que ofrezca a las personas afectadas una reparación por la violación del Convenio declarada en la presente sentencia con respecto a los demandantes». "Él [Tribunal Europeo] estima que el Estado demandado debería, ante todo, suprimir cualquier obstáculo a la obtención de una indemnización razonable con respecto al valor del bien expro- 
La Gran Sala hace explícita, sobre todo, esta nueva extensión en el asunto Hutten-Czapska contra Polonia de 19.6.2006 ${ }^{20}$. En este asunto, el sistema de control de alquileres era susceptible de afectar a un número todavía más importante de personas (alrededor de cien mil propietarios y de seiscientos mil a novecientos mil inquilinos); las circunstancias de dicha causa revelaban la existencia de un problema sistemático subyacente ligado a un grave fallo en el orden jurídico interno, a saber, la "disfunción de la legislación polaca en materia de vivienda, que imponía y continúa imponiendo a los propietarios particulares restricciones al aumento del alquiler de sus viviendas". Y prosigue el Tribunal: «pese a que el número de demandas similares pendientes ante el Tribunal sea actualmente de dieciocho -lo que, en comparación con el asunto Broniowski, puede parecer desdeñable-, una de esas demandas ha sido formulada por una asociación que integra a doscientos propietarios que denuncian una violación de sus derechos individuales. En cualquier caso, para que una situación sea calificada de sistemática y justifique la aplicación del procedimiento de la sentencia piloto, no se precisa necesariamente que un número determinado de demandas similares esté pendiente. Cuando se trata de violaciones sistemáticas o estructurales, el flujo de asuntos susceptibles de ser denunciados en el futuro ante el Tribunal Europeo es también un elemento importante que éste debe tomar en consideración con el fin de prevenir la saturación de su cometido a causa de asuntos repetitivos, lo que perturba el tratamiento eficaz de otros asuntos referentes a violaciones, a veces graves, de los derechos cuyo respeto el propio Tribunal tiene la tarea de asegurar» (§ 236). El Tribunal rechaza asimismo el argumento del Gobierno según el cual el procedimiento de la sentencia piloto ya no procedía tras una sentencia del Tribunal Constitucional en 2005, por cuando el Derecho interno todavía no era compatible con las exigencias del Convenio. En dicho asunto, el ejercicio del derecho de demanda individual se aproximaba al ejercicio de un derecho de acción colectiva ${ }^{21}$. La determinación del daño moral (de treinta mil euros) por el Tribunal tiene precisamente en cuenta el hecho de que la demandante ha debido soportar una fuerte presión psicológica y que ella «no se ha contentado con defender ante el Tribunal sus propios derechos, sino que

piado, garantizando así mediante medidas legales, administrativas y presupuestarias apropiadas la realización efectiva y rápida del derecho en cuestión con relación a otros demandantes afectados por bienes expropiados" ( $\$ 236 \& 237$ ).

20 Que confirma la sentencia de la Sección cuarta en el mismo asunto de 22.2.2005, yendo en cambio bastante más lejos en cuanto a la indicación de medidas generales.

${ }^{21}$ Véase el voto particular del juez Zupancic. 
también se ha tomado la molestia - en cierta medida al menos- de actuar en nombre de los propietarios que se encontraban en la misma situación que ella».

En lo que atañe a la indicación de medidas generales, el Tribunal, pese a expresarse de modo bastante amplio en sus afirmaciones (en razón de las dificultades propias de la identificación de las medidas generales adecuadas y del principio de libertad de medios del Estados), aprueba expresamente "las medidas mencionadas por la Corte Constitucional en sus recomendaciones de junio de 2005, que trazaban las grandes líneas de un mecanismo destinado a establecer un equilibrio entre los derechos de los propietarios y los de los arrendatarios, y exponían los criterios que deben servir para definir lo que son un alquiler de base, un alquiler económicamente justificado y un beneficio correcto». La identificación del problema estructural y el requerimiento al Estado para adoptar medidas generales son igualmente reproducidos en la parte dispositiva de la sentencia, lo mismo que había sucedido anteriormente en los asuntos Broniowski, Lukenda y Xenides-Arestis.

\section{La generalización del requerimiento al Estado para adoptar las medidas tendentes a la ejecución de las sentencias}

Durante varios años, el Tribunal Europeo había multiplicado las excepciones a la regla sagrada de la prohibición de indicar al Estado las medidas a adoptar a título de ejecución de las sentencias. El Tribunal se había mostrado más propicio a la hora de multiplicar esas excepciones en el ámbito de las medidas individuales. Los litigios referentes al derecho de propiedad han constituido el terreno elegido para las primeras excepciones; la petición de restitución del bien, por lo demás, adquiere un valor más vinculante desde el momento en que figura en la parte dispositiva de la sentencia ${ }^{22}$. Más frecuentemente, el Tribunal recomienda asimismo, cada vez en más asuntos, la reapertura de los procedimientos judiciales internos, cuando entiende que con ello se procede al restablecimiento más apropiado del derecho o derechos vulnerados $^{23}$. El contencioso más ilustrativo en este terreno es el del en-

22 TEDH, sentencia de 19.10.2006, Raicu contra Rumania, Sección tercera: el Tribunal Europeo requiere al Estado para que restituya el apartamento, previendo en su defecto la suma correspondiente a pagar.

23 TEDH, sentencia de 19.10.2006, Abdullah Altun contra Turquía, Sección tercera, § 38 (relativo a una violación del artículo 6.1) ; TEDH, sentencia de 19.10.2006, Majadallah contra Italia, Sección primera [violación del artículo 6.1 y 3.d)]; TEDH, 
juiciamiento de individuos ante tribunales que no respeten el principio de independencia o de imparcialidad ${ }^{24}$. Pero el Tribunal ha precisado que esta reapertura debe contar con el acuerdo de la propia víctima y desarrollarse ante un tribunal que respete las garantías del artículo $6.1^{25}$. A tal efecto conviene mencionar el supuesto particular de la sentencia Kounov contra Bulgaria, en donde el Tribunal Europeo toma como base la combinación de los artículos 46 y 41 para efectuar semejante recomendación ${ }^{26}$. En todos los casos, se trata de una invitación, puesto que el Tribunal Europeo rehúsa imponer al Estado una reapertura a petición del demandante, y menos aún exigir al Estado que reforme su Derecho interno para incorporar la posibilidad de reapertura ${ }^{27}$. Ello no obstante, cuando la reapertura es posible en Derecho interno, el Tribunal en ocasiones sí ha mencionado la recomendación de dicha medida, incluso en la parte dispositiva de la sentencia con el fin de ejercer presión sobre las autoridades estatales ${ }^{28}$.

Este tipo de recomendaciones se extiende ahora a las medidas generales, y la técnica de las sentencias piloto ha abierto la vía a la generalización del poder de recomendación del Tribunal y a su extensión sobre todo en el ámbito de las medidas generales.

Así, lo que en el estadio actual importa exclusivamente es la constatación de un fallo general (normativo o en la práctica) susceptible de

sentencia de 13.04.2006, Zentar contra Francia, § 35 [violación del artículo 6.1 y 3.d)]. Sobre todo, TEDH, sentencia de 1.3.2006, Sejdovic contra Italia, Gran Sala, § 126 (derechos de defensa).

24 TEDH, sentencia de 12.05.2005, Öcalan contra Turquía, Gran Sala, § 210.

$25 \mathrm{TEDH}$, sentencia de 2.2.2006, Duran Sekin c/Turquie, Sección tercera, § 45.

26 TEDH, sentencia de 23.5.2006, Kounov contra Bulgaria, Sección quinta, referente a la violación del artículo 6.1, tras haber sido condenado el individuo en rebeldía. En el asunto R.R. contra Italia de la Sección tercera de 9.6.2005, el fundamento viene constituido sólo por el artículo 46 , siendo posible en este supuesto la reapertura en el Derecho interno en caso de condena en rebeldía.

${ }_{27}$ TEDH, sentencia de 18.7.2006, Hostein contra Francia, Sección segunda, § 49: «En lo que concierne a la petición del demandante relativa a la introducción en Derecho interno de un mecanismo de revisión en materia civil, el Tribunal recuerda que semejante derecho no está garantizado como tal por el Convenio".

${ }_{28}$ TEDH sentencia de 26.1.2006, Lungoci contra Rumania, Sección tercera: " Declara a) que el Estado demandado debe asegurar, dentro de los seis meses a partir del día en que la sentencia sea definitiva, conforme al artículo 44.2 del Convenio, y si la parte demandante lo deseara, la reapertura del procedimiento, y que aquél debe simultáneamente pagarle 5000 (cinco mil) euros por daño moral, más cualquier otro montante que proceda a título de impuesto, a convertir en la moneda rumana según el cambio aplicable en la fecha de su pago". 
acarrear un contencioso cuantitativamente importante. En esto, los asuntos pilotos no constituyen realmente una novedad ${ }^{29}$; desde la sentencia Marckx, había sido asumido que las sentencias del Tribunal Europeo, desde el momento en que la violación tenía por fundamento una norma general, surtían efectos más allá de la víctima, podían generar un contencioso importante y los Estados estaban obligados a adoptar medidas generales. Lo que es nuevo es el uso (el descubrimiento) por el Tribunal Europeo del artículo 46 para indicar claramente al Estado la existencia de una deficiencia semejante y para ayudarle a delimitar los remedios que pongan fin a la misma pidiéndole que adopte medidas generales respecto de las cuales el propio Tribunal Europeo define sus contornos de manera más o menos clara. Los asuntos Tekin Yildiz contra Turquía de 10.11.2005 (Sección tercera) y Sürmeli contra Alemania de 8.6.2006 (Gran Sala) constituyen la ilustración de la generalización de esta política, en ausencia de mención de problema alguno a gran escala que pueda generar un contencioso masivo. En el último asunto mencionado, referente a la violación del artículo 13 del Convenio, el Tribunal Europeo evoca el proyecto de ley de un recurso por inactividad o carencia respecto del cual valora su carácter preventivo, y "anima a la adopción rápida de una ley que retome las propuestas del proyecto de ley en cuestión. El Tribunal estima por ello que no es necesario indicar medidas generales a adoptar a nivel nacional y que se impondrían en el marco de la ejecución de la presente sentencia» (párrafo 139). Si semejante proyecto no hubiera existido, podemos entonces deducir que el Tribunal Europeo habría indicado medidas generales a adoptar. En el asunto Tekin Yildiz, la Sección tercera del Tribunal Europeo había identificado las deficiencias del mecanismo oficial de peritaje médico forense en Turquía. Sin calificar las deficiencias nacionales de "estructurales", el Tribunal había estimado útil subrayar que se había "empleado a fondo al acercarse a las circunstancias comúnmente denunciadas en los cincuenta y tres asuntos que han sido objeto de la misión de investigación" (§ 91). Ciertamente, esta posición no es inédita en la jurisprudencia del Tribunal Europeo; pueden hallarse ejemplos incluso bastante alejados en el tiempo en los que el Tribunal se ha pronunciado sobre un proyecto de ley en tramitación, o ha asistido más directamente al Estado para que delimite las medidas a adoptar.

${ }^{29}$ Veáse SUNDBERG, F.: "L'effectivité des recours internes suite à des arrêts pilote", en G. Cohen-Jonathan, J.F. Flauss, y E. Lambert Abdelgawad (dir.), De l'effectivité des recours internes dans l'application de la Convention européenne des droits de l'homme, Bruylant, Nemesis, Coll. Droit et Justice n. ${ }^{\circ} 69,2006$, pp. 259-275, p. 260. 
Sin embargo, lo que era excepcional en el pasado ${ }^{30}$, pareciere en la actualidad responder a una política más sistemática, sobre la base del artículo 46. Bien es cierto que la técnica de la sentencia piloto alimenta esa predisposición del Tribunal consistente en indicar, de modo menos excepcional que en el pasado, las medidas generales a adoptar con el fin de asegurar una ejecución rápida y efectiva de las sentencias ${ }^{31}$, aun cuando la obligación de tomar medidas generales no figure en la parte dispositiva de la sentencia.

Esta nueva política del Tribunal no deja de suscitar algunas críticas, dado que a buen seguro no se corresponde con los poderes expresamente atribuidos a aquél en el texto del Convenio. En el asunto de la Gran Sala Hutten-Czapska contra Polonia, el juez Zagrebelsky, que no niega los efectos erga omnes de las sentencias del Tribunal Europeo, se muestra no obstante inquieto ante el hecho de que la técnica de la sentencia piloto rompa el equilibrio entre el Tribunal Europeo y el Comité de Ministros, y que ello pueda provocar un equivocado desplazamiento del Tribunal hacia el terreno político (más aún en ese asunto, que implicaba como medida general la reescritura del derecho de propiedad) ${ }^{32}$. La posición del Gobierno italiano, ante la Gran Sala en el asunto Sejdovic, merece igualmente ser objeto de atención, en tanto que bien podría ser suscrita por otros Estados. Según dicha argumentación, si «él no se opone, en principio, a que el Tribunal dé indicaciones bastante precisas sobre las medidas de carácter general a tomar», «sin embargo, la nueva práctica seguida por el Tribunal comporta el riesgo de reducir a la nada el principio de libertad de los Estados en la elección de las medidas de ejecución de las sentencias. El Tribunal,

30 Obsérvese, pese a todo, la tradicional prudencia del Tribunal Europeo en el asunto Tekin Yildiz, mencionando que las indicaciones de las medidas generales son "excepcionales" (§ 91).

31 Para una defensa de esta nueva política del Tribunal Europeo, léase LEACH, P.: "Beyond the Bug River - a new Dawn for redress before the european court of human rights?", (2005) EHRLR, issue 2, pp.148-164.

32 Véase la respuesta dada por el juez Zupancic en un voto particular sobre este mismo asunto: "Con el fin de respetar el espíritu del Convenio, tomemos en serio esas vacilaciones políticas y centrémonos en la cuestión siguiente: ¿Es mejor para Polonia ser condenada por el Tribunal Europeo en 80000 ocasiones y tener que pagar todos los gastos y costas procesales determinadas en esos 80000 asuntos, o resulta preferible decirle a este país: "jMiren, ustedes tienen que resolver una grave problema y preferimos que lo resuelvan en su casa...! Si ello puede ayudarles, vamos a indicarles cuáles son para nosotros las normas mínimas a tomar en consideración a tal efecto.. "? ¿Cuál de estas dos soluciones es más respetuosa con la soberanía nacional?». 
por lo demás, contradiría el espíritu del Convenio en ausencia de una base jurídica clara». Según el Gobierno italiano, esta interpretación viene confirmada por el Protocolo núm.14 y por una lectura literal de la resolución del Consejo de Ministros ${ }^{33}$. $Y$ añade que "de todas formas, si la práctica consistente en indicar medidas de carácter general debiera mantenerse, debería ser institucionalizada al menos en el Reglamento del Tribunal, o bien en las preguntas que el Tribunal formula a las partes, con el fin de permitir a éstas que presenten sus observaciones sobre la cuestión relativa a la naturaleza 'estructural' de la violación" (§§ 115-118).

Estas críticas, de hecho, ponen de manifiesto que la técnica de las sentencias piloto constituye algo más que un instrumento anodino tendente a acelerar la ejecución de las sentencias; revela, más bien, cambios de la mayor importancia en el contencioso europeo sobre derechos humanos.

\section{LA TÉCNICA DE LAS SENTENCIAS PILOTO: UN MECANISMO DE PRIMER ORDEN DE TRANSFORMACIÓN DE LA MISIÓN DEL TRIBUNAL}

La técnica de las sentencias piloto va acompañada de una prioridad que en el estadio actual concede el Tribunal Europeo al artículo 46 con respecto al artículo 41; va a la par igualmente de las transformaciones del cometido del Tribunal Europeo instauradas mediante la reforma del Protocolo núm. 14.

33 «117. En opinión del Gobierno, este reparto de competencias viene confirmado por el artículo 16 del Protocolo núm. 14 que, al modificar el artículo 46 del Convenio, introduce dos nuevos recursos: el recurso para interpretación y el recurso por infracción. Según el informe explicativo, el primero tendría por objeto «permitir al Tribunal Europeo que ofrezca una interpretación de una sentencia, y no de pronunciarse sobre las medidas adoptadas por la Alta Parte Contratante para cumplir la sentencia». En cuanto al segundo, se especifica que, en caso de que el Tribunal Europeo constate una violación, debe remitir el asunto al Comité de Ministros "con el fin de que éste examine las medidas a tomar". En fin, en su Resolución Res(2004)3, el Comité de Ministros invitó al Tribunal «a identificar en sus sentencias los eventuales problemas estructurales subyacentes, pero no a indicar igualmente las soluciones apropiadas. El reparto de competencias entre el Comité de Ministros y el Tribunal Europeo, tal como fue prevista por los autores del Convenio, por tanto no habría sido modificado". 


\section{La depreciación de la misión del Tribunal en virtud del artículo 41}

1.1. De la afirmación por el Tribunal de la prioridad del artículo 46 sobre el artículo $41 \ldots$

Las tres sentencias contra los Países Bajos de julio de 2006 referentes a los cacheos de detenidos habrían podido pasar desapercibidas, si el Tribunal Europeo no hubiera aprovechado la ocasión para emitir afirmaciones inesperadas ${ }^{34}$. En efecto, el Tribunal Europeo empieza ciertamente por subrayar el carácter inédito de la situación, puesto que los demandantes habían iniciado un procedimiento nacional para obtener una compensación equitativa en razón de la violación del Convenio Europeo, incluso antes de que el Tribunal Europeo hubiera dictado su sentencia sobre el fondo. El Tribunal Europeo establece como principio que él no puede permitir la coexistencia de dos procedimientos paralelos (uno a nivel interno, el otro ante el propio Tribunal Europeo) con el mismo objeto. Poco importa a este respecto que el procedimiento interno se encuentre ya pendiente en el momento en que la demanda fue presentada ante el Tribunal Europeo o que se haya recurrido antes ante dicho Tribunal, pese a que, en virtud artículo 35.1, la demanda sería declarada inadmisible en el primer supuesto mencionado. Para zanjar la cuestión, el Tribunal Europeo considera que debe retomar la idea del carácter subsidiario del Convenio. A continuación, el propio Tribunal examina su misión y deduce del artículo 41 que el otorgamiento de una compensación equitativa no constituye un elemento central de su misión, tratándose más bien de una función incidental. Según el Tribunal Europeo, el artículo 46 tiene así un objeto de mayor alcance que el artículo 41. Recuerda asimismo que el Estado sigue siendo libre para conceder una indemnización suplementaria respecto a las medidas de reparación obtenidas a nivel europeo. En el caso de autos, el Tribunal Europeo considera que la naturaleza de la violación precisa de una reparación pecuniaria. Con anterioridad, y con la finalidad de zanjar la cuestión bajo el ángulo del artículo 41, el Tribunal Europeo decide examinar previamente las consecuencias deducibles del artículo 46. Se trata en este caso de un asunto repetitivo respecto a los contenciosos Van der Ven y Lorsé y otros, referente a una práctica que ha afectado a todos los detenidos hasta el 1 de marzo de 2003; el Tribunal Europeo señala que se imponían sin lugar a dudas medidas generales, y que éstas debían comportar un mecanismo tendente a la

34 TEDH, sentencia de 6.7.2006, Baybasin contra Países Bajos, Khalid Salah contra Países Bajos, Salah contra Países Bajos, Sección tercera. 
compensación del daño sufrido por las víctimas de los cacheos. El Tribunal Europeo constata que la práctica ha sido abolida y que están abiertas causas civiles para indemnizar a los detenidos. Dada, pues, la existencia de recursos en vía civil formulados por los demandantes, el Tribunal Europeo concluye que el asunto no se presta a ser resuelto en el terreno del artículo 41, puesto que el Tribunal estima que deberá tener en cuenta la suma eventualmente otorgada en el ámbito interno antes de pronunciarse él mismo ${ }^{35}$. Lógicamente, lo deseable en estos asuntos sería encaminarlos hacia un arreglo amistoso.

La argumentación del Tribunal, sin lugar a dudas convincente, reviste a mayor abundamiento un carácter interesante e inédito por la argumentación finalista que ofrece del artículo 41 a la luz de las exigencias del artículo 46, así como por la prioridad otorgada al artículo 46 en los asuntos repetitivos. Se retoma igualmente una interpretación más literal del artículo 41, defendida por la doctrina mayoritaria, de conformidad con el principio de subsidiariedad del sistema europeo. Desde hace poco, se asiste así a un cambio de tendencia bastante espectacular en la jurisprudencia del Tribunal Europeo, en la medida en que en materia de seguimiento de la sentencia, la cuestión central, incluso para el propio Tribunal Europeo, ya no es el otorgamiento de una reparación pecuniaria. Lejos de haberse llegado a poner en tela de juicio el carácter insustancial del contencioso referente a la indemnización, seguramente se haya franqueando una nueva etapa en la materia.

\section{2. ...A la declaración de incompetencia del Tribunal Europeo a favor de los jueces internos en materia de compensación equitativa}

Esta jurisprudencia de julio de 2006, ¿ponía las bases para un cambio más radical consistente en inhibirse el Tribunal Europeo en provecho de los órganos jurisdiccionales nacionales en el otorgamiento de la compensación equitativa? El caso es que el informe del Grupo de Sabios de noviembre de 2006 no dudó en franquear ese estadio para proponer que, como regla general, la fijación del montante de la indemnización "se traslade al Estado afectado" (§ 96), en un plazo determinado y conforme a los criterios establecidos por la jurisprudencia eu-

35 Para una mayor ilustración sobre este aspecto, véase TEDH, sentencia de 19.10.2006, Tomasi contra Croacia, Sección primera, relativa a la violación del artículo 6.1. En virtud del artículo 41, el Tribunal Europeo tiene en cuenta la suma concedida por el Tribunal Constitucional. 
ropea. Se preconizaba una derogación a dicha regla «si semejante decisión se revelara necesaria con el fin de asegurar una protección efectiva de la víctima, especialmente en situaciones caracterizadas por una urgencia particular» ( $\$ 96$ ). Dicha fijación quedaría en manos de una instancia judicial única en cada Estado. Por otra parte, el Grupo de Sabios previó una posibilidad para la víctima de recurrir ante el Tribunal Europeo "para contestar la decisión nacional a la luz de dichos criterios o cuando el Estado no respetara el plazo que le hubiera sido concedido para fijar el montante de la indemnización» (§ 99). Esta propuesta no resulta sin embargo convincente: la probabilidad de divergencias entre Estados es alta, a lo que se añade el riesgo de una infracción por parte del Estado a la hora de fijar el montante a satisfacer. La imagen del sistema europeo quedaría considerablemente empañada a los ojos de unas víctimas que estarían constantemente desconcertadas entre el nivel europeo y el nivel nacional. Así pues, es casi seguro que, al final, ello no se saldará con una ganancia real de tiempo, sino con un contencioso suplementario que bloqueará al Tribunal Europeo. Las propuestas contenidas en el Informe de Lord Woolf no iban tan lejos, al prever el establecimiento en la Secretaría del Tribunal de una «unidad artículo 41 » encargada de elaborar líneas directrices sobre las indemnizaciones para facilitar la tarea de los jueces, e incluso de una "guía referente al montante de las indemnizaciones concedidas por el Tribunal Europeo" ${ }^{36}$. Pero el informe, de cuyo campo de estudio quedaba excluida toda medida que comportara una modificación del texto del Convenio, preveía que «nada impide al Tribunal convenir con un Estado miembro que corresponderá a éste resolver las cuestiones sobre una indemnización», y, con tal proceder, "suscribir acuerdos similares con los diferentes Estados miembros ${ }^{37}$.

Estas propuestas se inscriben incontestablemente en la tendencia afirmada en la jurisprudencia más reciente del Tribunal Europeo tendente a otorgar prioridad al artículo 46 con respecto al artículo 41, así como en los esfuerzos del Tribunal Europeo conducentes a delimitar criterios objetivos de cálculo de la satisfacción equitativa. Es eso lo que ya ha ocurrido en materia de contencioso relativo a la duración excesiva del procedimiento en virtud del artículo 6.1. En algunos asuntos concernientes a Italia, de fecha 10.11.2004, especialmente en el caso Cocchiarella contra Italia, la Sección primera del Tribunal Europeo había es-

36 Esta unidad del artículo 41 ha sido establecida en otoño de 2006.

37 Etude des méthodes de travail, Cour européenne des droits de l'homme, diciembre 2005, The Right Honorable The Lord Woolf, p. 39. 
tablecido "criterios particulares" relativamente precisos, extraídos de estudios llevados a cabo por la Secretaría del Tribunal ${ }^{38}$.

El Tribunal Europeo desea así imprimir mayor transparencia a su jurisprudencia, con el fin de dotar también de mayor coherencia a ésta; desea asimismo mostrar que este tipo de contencioso, bastante técnico, podría, siguiéndose dichos criterios, ser transferido sin demasiada dificultad a los órganos jurisdiccionales internos.

\section{Centrarse en el contencioso de mayor entidad mediante la limitación del derecho al recurso individual}

Para el Tribunal Europeo, el centrar sus prioridades en el artículo 46, así como en la determinación de las medidas generales y en la resolución de los asuntos repetitivos, parece anunciar una evolución de su

38 «2. Criterios particulares respecto al daño moral

1. En lo que concierne a la evaluación equitativa del daño moral sufrido en razón de la duración de un procedimiento, el Tribunal Europeo estima que una suma de entre 1000 y 1500 euros por año de duración del procedimiento (y no por año de retraso) constituye una base de partida para el cálculo a efectuar. El resultado del procedimiento nacional (tanto si la parte demandante pierde, gana o acaba concluyendo un arreglo amistoso) no reviste importancia como tal al ponderar el daño moral sufrido por el hecho de la duración del procedimiento.

El montante global ascenderá a 2000 euros si la controversia presenta implicaciones importantes, especialmente en materia de derechos laborales, de estado y capacidad de las personas, de pensiones, de procedimientos particularmente graves en relación con la salud o la vida de las personas.

El montante de base quedará reducido a tenor del número de órganos jurisdiccionales que tuvieron que resolver durante la duración del procedimiento, del comportamiento de la parte demandante —especialmente del número de meses o de años vinculados con recursos no justificados imputables a la parte actora-, del alcance del litigio - por ejemplo, cuando el alcance patrimonial es poco importante para la parte recurrente-, y en función del nivel de vida del país. Puede asimismo afrontarse la posibilidad de una reducción cuando el demandante haya participado de forma breve en un procedimiento que después ha continuado como heredero.

Dicho montante podrá quedar igualmente minorado cuando la parte demandante ya hubiera obtenido en el ámbito nacional una declaración de violación y una suma de dinero en el marco de una vía interna de recurso. Además del hecho de que la existencia de una vía de recuso en el plano interno se adecua plenamente al principio de subsidiariedad propio del Convenio, dicha vía de recurso es más próxima y accesible que el recurso ante el Tribunal, es más rápida, y se ejerce en la lengua de la parte demandante; por tanto, esa vía presenta ventajas que conviene tomar en consideración". 
misión que prepara el Protocolo núm. 14 intentando limitar el derecho al recurso individual.

De conformidad con el principio de subsidiariedad, el Tribunal Europeo ha intervenido tradicionalmente para sancionar las deficiencias del nivel interno que las autoridades nacionales habrían debido rectificar con motivo del ejercicio de los recursos nacionales. Pero, en razón de la explosión del contencioso, parece que el Tribunal Europeo ya no cuente con los medios para hacer frente a semejante ambición. Con ocasión de los trabajos realizados en vista a la adopción del Protocolo núm. 14, se formularon diversas propuestas con objeto de ofrecer al Tribunal Europeo la posibilidad de seleccionar el contencioso y de rehusar el enjuiciamiento de algunos asuntos. La primera fórmula sometida por el Grupo de Evaluación preveía el rechazo de las demandas «que no plantearan ninguna cuestión sustancial a la luz del Convenio" ${ }^{39}$; la apreciación por el Tribunal Europeo sobre qué deba entenderse por "una cuestión sustancial» tendría en cuenta "especialmente la situación predominante en el Estado demandado, así como la existencia de vías de recurso internas efectivas". Sin embargo, el Grupo consideraba que semejantes asuntos declarados inadmisibles deberían ser "reexaminados" por los órganos jurisdiccionales nacionales. Tras un cierto número de críticas que expresaban su temor a una limitación abusiva del derecho de recurso individual, la fórmula por la que finalmente se optó fue modificada en diversas ocasiones por los expertos gubernamentales y se revela menos restrictiva, incorporando una propuesta austriaca tendente a preservar la subsidiariedad del mecanismo europeo: «El apartado 3 del artículo 35 del Convenio queda redactado como sigue: 3. el Tribunal declarará inadmisible cualquier demanda individual introducida en virtud del artículo 34 cuando estime: a) (...) b) que el demandante no ha sufrido ningún perjuicio importante, salvo en caso de que el respeto de los derechos humanos garantizados por el Convenio y sus Protocolos exija un examen sobre el fondo de la demanda y siempre que no se rechace por tal motivo ningún asunto que no haya sido debidamente examinado por un tribunal interno". Es cierto que «(...) una limitación drástica del alcance del recurso individual sólo unos años más tarde de la entrada en vigor del Protocolo núm. 11 pre-

39 Proposición del Grupo de Evaluación de 2001 (EG-Court(2001)1, informe de 27.9.2001), apartado 93. Según el Comité Director de Derechos Humanos, esta propuesta acarrearía "una importante limitación del derecho de recurso individual que, por razones jurídicas y prácticas, no podría ser compensada por un sistema según el cual el Tribunal Europeo pudiere remitir los asuntos rechazados a las jurisdicciones nacionales o a otras autoridades para una nueva decisión". 
sentaría el riesgo de tener un impacto jurídico, pero también político y simbólico, muy importante ${ }^{40}$.

A ello debe añadirse que algunos Estados se oponían totalmente a poner en tela de juicio el derecho individual de recurrir, opinión igualmente defendida por la mayoría de la doctrina ${ }^{41}$. Por lo demás, resulta pertinente mencionar la existencia de una disposición transitoria en cuanto a su puesta en marcha: "en los dos años siguientes a la entrada en vigor del presente protocolo, únicamente las Salas y la Gran Sala del Tribunal podrán aplicar el nuevo criterio de admisibilidad ${ }^{42}$. Se revela ciertamente esencial que esta condición, de perfiles un tanto difusos, sea precisada desde el primer momento por las Salas y la Gran Sala.

Según el Comité Director de Derechos Humanos, el tipo de asuntos que pueden verse afectados es el siguiente: los «asuntos referentes a la duración de los procedimientos civiles sobre cuestiones cuyo interés material sea menor de 500 euros; los litigios de vecindad; una controversia en torno al lugar en el que debería ser pagada una pensión de jubilación", esto es, "alrededor del $5 \%$ de los asuntos actualmente admisibles". Sin embargo, esta propuesta adolece de puntos débiles: es difícil identificar qué es un "perjuicio importante". Además, "la ganancia en términos de tiempo y de productividad para el Tribunal Europeo residiría esencialmente en la ausencia de motivación, lo que le conferiría un amplio poder discrecional», lo cual no sería «ni razonable

40 SICILIANOS, L-A.: "La 'réforme de la réforme' du système de protection de la Convention européenne des droits de l'homme ", AFDI, 2003, pp. 611 ss., p. 626.

41 BENOIT-ROHMER, F.: "ll faut sauver le recours individuel...", Rec. Dalloz, n. ${ }^{\circ}$ 38, 30.10.2003, crónica pp. 2584 ss. WADHAM, J., \& SAID, T.: «What price the right of individual petition: report of the evaluation group to the Committee of Ministers on the European Court of Human Rights", EHRLR, issue 2, 2002, pp. 169 ss., p.170. COHEN-JONATHAN, G.: "Conclusions", pp.125 ss., in La réforme de la Cour européenne des droits de I'homme, COHEN-JONATHAN, G., \& PETTITI, Ch. (éd.), Bruylant, Coll. Droit et Justice, n. ${ }^{\circ}$ 48, 2003. Para una apreciación más matizada, SICILIANOS, L.A.: "L'objectif primordial du Protocole n. ${ }^{\circ} 14$ à la CEDH: alléger la charge de travail de la Cour", in COHEN-JONATHAN, G., \& FLAUSS, J.F.: La réforme du système de contrôle contentieux de la CEDH (Le Protocole n. 14 et les Recommandations et Résolutions du Comité des Ministres), Nemesis, Bruylant, 2005, pp. 55 ss. Para un "voto particular», cfr. FLAUSS, J.F.: "La réforme de la réforme - Propos conclusifs sous forme d'opinion séparée", in COHEN-JONATHAN, G., \& FLAUSS, J.F.: La réforme du système de contrôle contentieux de la CEDH (Le Protocole n. 14 et les Recommandations et Résolutions du Comité des Ministres), Nemesis, Bruylant, 2005, pp. 179 ss.

42 Artículo 20, apartado 2, del Protocolo núm. 14.

(C) UNED. Revista de Derecho Político 
ni oportuno ${ }^{43}$. Por otra parte, el sistema actual, en el estadio de la admisibilidad ya es muy selectivo (mediante la interpretación estricta de la cláusula "manifiestamente mal fundado") $)^{44}$. Desde un punto de vista exclusivamente cuantitativo, el beneficio sería extremadamente limitado, y no podría aplicarse a los asuntos repetitivos. Por añadidura, "la nueva condición de admisibilidad propuesta desnaturalizaría el principio decisorio que gobierna el funcionamiento del Tribunal puesto que, con ello, una demanda 'manifiestamente bien fundada' o que contenga una probable violación del Convenio podría ser declarada inadmisible antes que admisible»" ${ }^{45}$. Asuntos "mínimos» en el pasado han propiciado el desarrollo de la función jurisdiccional del Tribunal Europeo ${ }^{46}$. Sobre todo, en el seno del Comité Director de Derechos Humanos, algunos expertos han puesto de relieve "el mensaje negativo a las jurisdicciones internas según el cual estas últimas podrían no ocuparse de algunas violaciones menores del Convenio. De manera más general, de ello podría igualmente deducirse que las jurisdicciones internas no se verían animadas a examinar los recursos relativos al Convenio con toda la atención necesaria»" "Si el Tribunal Europeo no ad-

$43 \mathrm{Y}$ «(...) si la necesidad de abordar el carácter satisfactorio del examen de una de las pretensiones formuladas a nivel nacional debiere de hecho conllevar la obligación de acometer una apreciación de las cuestiones de fondo en el estadio de la admisibilidad, ello iría totalmente contra el objetivo consistente en aligerar la carga de trabajo del Tribunal Europeo" (CDDH-GDR(2004)001 Rev, Respuesta del TEDH, apartado 19). El Tribunal Europeo proponía una fórmula extremadamente vaga: "cuando el respeto de los derechos humanos garantizados por el Convenio y sus Protocolos no imponga el examen de la demanda".

44 PETTITI, Ch.: "Rapport», in La réforme de la Cour européenne des droits de I'homme, COHEN-JONATHAN, G., \& PETTITI, Ch. (éd.), Bruylant, Coll. Droit et Justice, n. ${ }^{\circ} 48,2003$, pp. 87 ss., pp. 95-96: los abogados franceses con experiencia en Estrasburgo se oponen, en un $90 \%$, a cualquier restricción al derecho de recurso, estimando que las condiciones de inadmisibilidad ya son de aplicación estricta, y parten de la constatación de una aplicación todavía imperfecta del Convenio Europeo de Derechos Humanos por parte del juez nacional; temen asimismo que el juez nacional no modifique su jurisprudencia por existir un pequeño riesgo de control en Estrasburgo.

45 Anexo 3: "Pour le droit de recours individuel", por TULKENS, F., FISCHBACH, J., CASADEVALL, J., THOMASSEN, W., in La réforme de la Cour européenne des droits de I'homme, COHEN-JONATHAN, G., \& PETTITI, Ch. (éd.), Bruylant, Coll. Droit et Justice, n. ${ }^{\circ} 48,2003$, pp. 171 ss.

46 WACHSMANN, P.: "Droit de recours individuel et protection de la fonction jurisprudentielle de la Cour “" $R U D H, 2002$, pp. 309 ss., pp. 310-311. SUDRE, F.: Droit européen et international des droits de l'homme, PUF, 7. ${ }^{a}$ ed., 2005, p. 590.

$47 \mathrm{CDDH}(2003) 026$ Addendum I Final, apartado 34. 
mite un asunto por estimar que éste no causa un perjuicio importante al demandante o no suscita una cuestión grave, ¿por qué deberían estar los Estados más atentos a la hora de velar por el respeto de los derechos del Convenio?» ${ }^{48}$.

Es demasiado pronto todavía para conocer la interpretación que de todo esto hará el Tribunal Europeo, si amplia o estricta. Ello no obstante, esta reforma presenta el riesgo de proyectar consecuencias negativas: como quiera que sólo el $5 \%$ de los asuntos actualmente admisibles podrían verse afectados, "esto quiere decir que la solución propuesta no resuelve el problema a más o menos largo plazo, y que se revelará necesario abordarlo nuevamente. Pero, como se habrá limitado el recurso individual mediante una primera reforma, quedará ampliamente expedita la vía para una restricción más consecuente ${ }^{49}$. Como ha subrayado ese mismo autor, "por último, y es lo que se presenta como más grave, restringir la admisibilidad de las demandas individuales nos lleva a no considerar el derecho al recurso individual como un derecho fundamental, o cuando menos a admitir tal vez demasiado fácilmente que ese derecho pueda sufrir restricciones ${ }^{50}$.

\section{Reforzar el carácter supremo del Tribunal a través de nuevos recursos}

El Protocolo núm. 14 ha establecido dos nuevos recursos ante el Tribunal de Estrasburgo, en provecho del Comité de Ministros. En un momento de saturación del órgano judicial, sólo razones de primer orden podrían justificar semejantes medidas. Simbólicamente, esas medidas disponen que el Tribunal deba ser el órgano supremo en materia de interpretación y de inejecución de sus propias sentencias.

48 Anexo 3: "Pour le droit de recours individuel», por TULKENS, F., FISCHBACH, J., CASADEVALL, J., THOMASSEN, W., in La réforme de la Cour européenne des droits de I'homme, COHEN-JONATHAN, G., \& PETTITI, Ch. (éd.), Bruylant, Coll. Droit et Justice, n. ${ }^{\circ}$ 48, 2003, pp.171 ss., pp. 174-175. BENOIT-ROHMER, F.: "Le Protocole 14 à la $\mathrm{CEDH}$ : une atteinte au droit de recours individuel devant la Cour européenne des droits de l'homme?", doc. Ronéo., IHEE de Estrasburgo, p. 4.

49 BENOIT-ROHMER, F.: "ll faut sauver le recours individuel...», Rec. Dalloz, n. ${ }^{\circ} 38,30.10 .2003$, crónica, pp. 2584 ss., p. 2587.

50 BENOIT-ROHMER, F.: "Le Protocole 14 à la CEDH : une atteinte au droit de recours individuel devant la Cour européenne des droits de I'homme?", doc. Ronéo., IHEE de Estrasburgo, p. 35. 
3.1. El recurso al Tribunal en caso de "dificultad de interpretación» de la sentencia

Según el nuevo artículo 46, apartado $3^{51}$, esta nuevo diseño del recurso ante el Tribunal Europeo viene a responder a un síntoma claramente identificado por la doctrina y por los órganos del Consejo de Europa: la ausencia de legibilidad de la sentencia es perjudicial a veces para su correcta y rápida ejecución.

Es interesante remontarse, siquiera brevemente, a la historia de la elaboración de esta nueva disposición, para comprender mejor su verdadero alcance. Por temor a poner en entredicho la calidad del trabajo de los jueces y la separación de las misiones encomendadas al Comité de Ministros y al Tribunal Europeo, no se trataría más que de establecer inicialmente una simple "información» al Tribunal Europeo por parte del Comité de Ministros ${ }^{52}$, que no exigiría una revisión del texto del Convenio. Adicionalmente, por temor a una deriva hacia la indicación por el Tribunal Europeo de los medios para ejecutar sus sentencias, fueron formuladas fuertes reticencias en este terreno al Comité de Ministros por el Grupo de Evaluación sobre el Tribunal Europeo de Derechos Humanos ${ }^{53}$, por el Presidente del Tribunal Europeo ${ }^{54}$, y por el Comité Director de Derechos Humanos en su Dictamen sobre la recomendación $1477(2000)$ de la Asamblea Parlamentaria relativa a la ejecución de las sentencias del Tribunal Europeo de Derechos Humanos, adoptado en noviembre de 2001. Incluso la Comisión de Venecia, en su Dictamen n. ${ }^{\circ} 209 / 2002$, emitió la opinión según la cual sería oportuno animar al Tribunal Europeo a indicar los medios para ejecutar sus sentencias:

51 "Cuando el Comité de Ministros estime que la supervisión de la ejecución de una sentencia definitiva se encuentre obstaculizada por una dificultad de interpretación de dicha sentencia, podrá acudir al Tribunal Europeo con el fin de que éste se pronuncie sobre dicha cuestión interpretativa. La decisión de acudir al Tribunal se adoptará por mayoría de dos tercios de representantes con derecho de voto en el Comité».

52 Cfr. CDDH-GDR(2001)010, 15.6.2001, informe de actividades, Anexo I, 2. ${ }^{\text {a }}$ reunión, abril 2001, punto 5, La Sugerencia A.iii.3 Ileva por título: "Informer la Cour de I'exécution des arrêts»/»/nformar al Tribunal de la ejecución de las sentencias": (apartado 27): "No se trata de otorgar un poder al Comité de Ministros respecto al trabajo del Tribunal Europeo, sino de informar al Tribunal de las dificultades que pueden sobrevenir con motivo de la fase de la ejecución de algunas de sus sentencias".

53 Documento EG Court(2001)1, de 27.9.2001, «Rapport du groupe d'évaluation au Comité des ministres sur la Cour européenne des droits de l'homme», apartado 49 . 
esta vía (apartado 61) "sería preferible respecto al otorgamiento al Comité de Ministros del poder de pedir formalmente la interpretación de una sentencia, como fue sugerido por la Asamblea Parlamentaria».

En consecuencia, no resulta sorprendente comprobar que el resultado final sea bastante modesto y se aproxime al procedimiento de interpretación clásico ya conocido. Sólo está legitimado en tal sentido para acudir al Tribunal Europeo el Comité de Ministros (careciendo en cambio de dicha facultad el Estado denunciado o el demandante) por mayoría de dos tercios, lo cual, según el informe explicativo, debería conducir a un "uso prudente de esta posibilidad que se le ofrece con el fin de no sobrecargar al Tribunal Europeo». No se establece ningún límite temporal, lo cual queda confirmado en el informe explicativo, pudiendo entonces surgir efectivamente la necesidad de interpretación mucho después de la fecha del pronunciamiento de la sentencia. Así las cosas, este procedimiento sólo debería afectar a casos bastante aislados en donde el Tribunal Europeo no hubiera tenido ocasión de precisar su jurisprudencia mediante una sentencia ulterior, o no hubiera indicado las medidas generales a adoptar dada su nueva "política» desde el asunto Broniowski.

3.2. El recurso al Tribunal en caso del incumplimiento de un Estado al ejecutar una sentencia

La introducción de un recurso por incumplimiento, mediante el artículo 46, apartados 4 y 5, del Convenio ${ }^{55}$, a semejanza de lo que existe en el marco de la Unión Europea, posee el valor de símbolo de un sistema regional que se pretende más vinculante y más autónomo, en el marco de una comunidad jurídica y política reforzada.

La legitimación en este ámbito sólo la ostenta el Comité de Ministros. Dicha legitimación no está abierta al individuo que sea víctima y parte en la sentencia: sin embargo, el derecho a la ejecución de una

54 CDL-AD (2002)34, 18.12.2002, Dictamen n. ${ }^{\circ}$ 209/2002, apartado 6.

55 «4. Cuando el Comité de Ministros estime que la Alta Parte Contratante rehúsa cumplir una sentencia definitiva en un litigio en el que aquella es parte, podrá, tras requerir a dicha Parte y mediante decisión adoptada por mayoría de dos tercios de los representantes con derecho de voto en el Tribunal, someter al Tribunal la cuestión relativa al respeto por dicha Parte de su obligación en virtud del apartado 1. 5. Si el Tribunal constatara una violación del apartado 1, remitirá el asunto al Comité de Ministros con el fin de que éste examine las medidas a tomar. Si el Tribunal constatara que no ha habido violación del apartado 1, remitirá el asunto al Comité de Ministros, que decidirá su archivo". 
sentencia es un elemento del derecho a un proceso equitativo según la jurisprudencia del Tribunal Europeo (asunto Hornsby contra Grecia, 1997 ${ }^{56}$. Ciertamente, las víctimas no han dudado a la hora de acudir nuevamente ante el Tribunal Europeo para que éste constate una violación continuada de sus derechos, en razón de la inejecución de una sentencia precedente que les había resultado estimatoria: las sentencias Mehemi (.$^{\circ}{ }^{2}$ ) contra Francia (10.4.2003), Wynne (.$\left.^{\circ}{ }^{2}\right)$ contra Reino Unido (16.10.2003) y Slimane-Kaïd $\left(n .^{\circ}\right.$ 2) contra Francia constituyen ejemplos manifiestos de ello. También se percibía un cierto riesgo de abuso por parte de los individuos.

La legitimación al efecto ya no está abierta al Estado denunciado, o al conjunto de los demás Estados según la lógica de garantía colectiva que fue un fracaso. El objetivo de este procedimiento consiste más bien en respaldar al Comité de Ministros en caso de oposición persistente de un Estado. La solemnidad de este acontecimiento viene marcada por el hecho de que el Tribunal Europeo actuará en composición de Gran Sala (nuevo artículo 31, letra b). El resultado del procedimiento es una sentencia del Tribunal Europeo, mediante la que no se trata en absoluto, como señala claramente el informe explicativo (apartado 99) «de reabrir ante el Tribunal la cuestión de fondo sobre la violación ya resuelta a través de la primera sentencia», sino de determinar si el Estado ha tomado las medidas requeridas a tenor de la sentencia de fondo condenatoria. Este recurso debería quedar reservado a algunos asuntos muy específicos, como la sentencia llascu y otros contra Moldavia y Federación de Rusia.

Así pues, estas dos nuevas modalidades de recurso debería verificarse raramente en la práctica, pero su finalidad es análoga: favorecer la ejecución de las sentencias especialmente para evitar una saturación ulterior del Tribunal Europeo, marcando así -incluso en materia de ejecución de sentencias - la supremacía del Tribunal (sobre el Comité de Ministros). De tal suerte, ¿el Tribunal Europeo no se aproximaría así, poco a poco, a un verdadero Tribunal Supremo?

\section{CONCLUSIÓN}

A través de la técnica de las sentencias piloto, el Tribunal Europeo de Derechos Humanos pretende, ciertamente, con absoluta buena fe,

56 En el mismo sentido, COHEN-JONATHAN, G.: "Garantir l'efficacité à long terme (...)", op. cit., RTDH, n. ${ }^{\circ}$ 56-2003, pp. 1146-1147. 
ayudar a los Estados y facilitar el trabajo del Comité de Ministros en el ámbito de la ejecución de las sentencias. Sin embargo, se sospecha que tras ese reto primordial inmediato se adivina, más o menos a largo plazo, un desafío de mayor envergadura: la voluntad, de los propios jueces, de transformar, a través de pequeños hitos sucesivos, su Tribunal (convertido a menudo en una instancia más de apelación - un cuarto nivel de jurisdicción- muy útil respecto de centenares de jurisdicciones nacionales) en un Tribunal Supremo que se centraría en algunos asuntos primordiales y serviría de guía última para las autoridades nacionales. El nivel de protección de los derechos humanos en Europa no debería necesariamente verse aquejado a causa de ello, siempre que, sin embargo, las autoridades nacionales se muestren dispuestas a seguir a su maestro. $Y$ no es del todo seguro que el conjunto de los Estados Partes en el Convenio puedan seguir esta nueva vía.

\section{Title}

The European Court of human rights and the pilot-judgment approach: a minor revolution is on in Strasbourg

\section{Contents}

I. Introduction. II. The pilot-judgment approach: a prima facie minor change to prevent another violation: 1. The Broniowski v/Poland case. 2. The pilot-judgment procedure is becoming more common and widespread. 3. The Court more often orders the State how to comply with the judgment. III. The pilot-judgment approach: a major lever to change the task of the Court: 1 . The task of the Court according to article 41 is discredited: 1.1. From the statement by the Court of the priority of article 46 over article $41 \ldots 1.2$. to the withdrawal of the European Court in relation to just satisfaction in favour of the national judges. 2. Focus on the most important cases by restricting the right to individual applications. 3. Strengthen the supreme court aspects of the European Court by adding new judicial actions: 3.1. the referral to the Court in case of a "problem of interpretation" of the judgment. 3.2. the referral to the Court in case of a State does not abide by a judgment. IV. Conclusion.

\section{Palabras clave}

Sentencias piloto; satisfacción equitativa, caso Broniowski contra Polonia; artículo $46 \mathrm{CEDH}$; artículo $14 \mathrm{CEDH}$; Protocolo núm. 14 al $\mathrm{CEDH}$; fuerza vinculante de las sentencias; casos repetitivos; violaciones sistemáticas. 


\title{
Key words
}

Pilot judgments; Just satisfaction; Case of Broniowski v/Poland; article 46 of the ECHR; article 41 of the ECHR; Protocol 14 to the ECHR; binding force of the judgments; repetitive cases; systematic violations.

\section{Resumen}

En el caso Broniowski contra Polonia, referente a un problema de compatibilidad con el Convenio Europeo de Derechos Humanos de un proyecto legislativo que afectaba a un gran número de personas (en torno a las 80.000), el Tribunal Europeo declaró por vez primera la existencia de una violación sistemática; de este modo, dejaba claro que podían imponerse medidas generales a nivel nacional para ejecutar una sentencia, y que dichas medidas debían tomar en consideración la gran cantidad de personas afectadas, así como remediar una deficiencia sistemática puesta de manifiesto por el Tribunal Europeo al constatar la correspondiente violación. El Tribunal Europeo observó asimismo que dichas medidas debían prever el modo de restablecer en sus derechos a las personas afectadas por la violación del Convenio Europeo.

Esta especie de nuevo enfoque del Tribunal Europeo al enjuiciar problemas sistemáticos o estructurales en el ordenamiento nacional, conocido como el "procedimiento de la sentencia piloto", ha sido adoptado otros casos desde 2004. Si el objetivo oficial de este nuevo enfoque radica en facilitar una más rápida y eficaz ejecución de las sentencias del Tribunal Europeo y, por ende, evitar el planteamiento de casos repetitivos ante él, esta nueva política consistente en indicar con mayor frecuencia las medidas generales a adoptar por el Estado es, según la autora, parte de un plan más general que apunta a la transformación del Tribunal Europeo de Derechos Humanos en un Tribunal Supremo; algunas de las medidas contenidas en el Protocolo núm. 14, cuya entrada en vigor se produce este mismo año 2007, así como la nueva prioridad conferida por el Tribunal al artículo 46 frente al artículo 41 del Convenio Europeo, revela alguno de esos cambios fundamentales que se están produciendo en Estrasburgo.

\begin{abstract}
In the case of Broniowski v. Poland, concerning the issue of the compatibility with the European Convention on Human Rights of a legislative scheme that affected a large number of persons (some 80,000 ), the European Court found for the first time the existence of a systemic violation thereby made clear that general measures at national
\end{abstract}


level were called for in execution of the judgment and that those measures should take into account the many people affected and remedy the systemic defect underlying the Court's finding of a violation. It also observed that they should include a scheme offering to those affected redress for the Convention violation.

This kind of adjudicative approach by the Court to systemic or structural problems in the national legal order, described as a "pilotjudgment procedure", has been adopted in several other cases since 2004. If the official aim of this new approach is to facilitate the most speedy and effective execution of its judgments of the European Court and so to avoid repetitive cases before it, this new policy, consisting in indicating more often the general measures to be adopted by the State, is, according to the author, part of a general plan to turn the European Court of human rights into a Supreme Court; some of the measures contained in Protocol 14, which should enter into force that year, as well as the new priority given by the Court to article 46 over article 41, reveal some of these fundamental changes in Strasbourg. 